Revista Brasileira de Cartografia

ISSN 1808-0936 | https://doi.org/10.14393/revbrascartogr

Sociedade Brasileira de Cartografia, Geodésia, Fotogrametria e Sensoriamento Remoto

\title{
Avaliação Laboratorial da Parametrização de Dados LST em Superfícies Planas
}

\section{Laboratory Evaluation of TLS Data Parameterization on Planes Surfaces}

\section{Samir de Souza Oliveira Alves ${ }^{1}$, Felipe Andrés Carvajal Rodríguez², Lívia Faria Sampaio ${ }^{3}$, Leandro Ítalo Barbosa de} Medeiros ${ }^{4}$, Luís Augusto Koenig Veiga ${ }^{5}$ e Ivandro Klein ${ }^{6}$

1 Universidade Federal do Paraná, Departamento de Geomática, Programa de Pós-Graduação em Ciências Geodésicas, Curitiba, Brasil. E-mail: samirsoalves@hotmail.com.

ORCID: https://orcid.org/0000-0003-3083-0681

2 Universidade Federal do Paraná, Departamento de Geomática, Programa de Pós-Graduação em Ciências Geodésicas, Curitiba, Brasil. E-mail: felipe.carvajalro@gmail.com.

ORCID: https://orcid.org/0000-0003-2418-3924

3 Universidade Federal do Paraná, Departamento de Geomática, Programa de Pós-Graduação em Ciências Geodésicas, Curitiba, Brasil. E-mail: liviafariasampaio@gmail.com.

ORCID: https://orcid.org/0000-0002-2543-5014

4 Universidade Federal do Paraná, Departamento de Geomática, Programa de Pós-Graduação em Ciências Geodésicas, Curitiba, Brasil. E-mail: leandro.medeiros@ufpr.br.

ORCID: https://orcid.org/0000-0002-0437-1269

5 Universidade Federal do Paraná, Departamento de Geomática, Programa de Pós-Graduação em Ciências Geodésicas, Curitiba, Brasil. E-mail: kngveiga@gmail.com.

ORCID: https://orcid.org/0000-0003-4026-5372

6 Instituto Federal de Educação, Ciência e Tecnologia de Santa Catarina, Departamento Acadêmico de Construção Civil, Curso Técnico de Agrimensura, Florianópolis, Brasil/ Universidade Federal do Paraná, Departamento de Geomática, Programa de PósGraduação em Ciências Geodésicas, Curitiba, Brasil. E-mail: ivandroklein@gmail.com.

ORCID: https://orcid.org/0000-0003-4296-592X

Resumo: O uso dos dados derivados dos levantamentos através do Laser Scanner Terrestre (LST) vem sendo investigado nas atividades de monitoramento geodésico de estruturas de engenharia como pontes, barragens e torres de concreto. A complexidade destes sistemas torna necessária a busca por abordagens que minimizem os erros, derivados das observações primárias dos ângulos e distâncias em cada ponto tridimensional, como a parametrização dos dados por elementos geométricos. Neste trabalho é apresentada uma avaliação laboratorial da estabilidade do processo de parametrização das nuvens de pontos por meio do ajustamento de observações em planos. Para a realização do experimento, desenvolveu-se um protótipo contendo uma superfície plana de movimentação controlada, sobre a qual se realizaram pares de varreduras com LST em variadas posições do plano. No processo de avaliação da estabilidade, utilizou-se o teste global do ajustamento entre os pares de varredura. Os resultados demonstraram que a estabilidade da parametrização está condicionada à relação posicional entre o plano e o LST no momento da varredura. Palavras-chave: Laser Scanner Terrestre. Parametrização. Teste Global.

\begin{abstract}
The use Terrestrial Laser Scanner (TLS) data has been investigated in geodetic monitoring activities of engineering structures such as bridges, dams and concrete towers. The complexity of these systems makes it necessary to search for approaches that minimize errors, which are derived from the primary observations of angles and distances in each three-dimensional point, such as the parameterization of data by geometric elements. This research presents a laboratory evaluation of the stability of the point cloud parameterization process by adjusting data in planes. To carry out the experiment, a prototype containing a controlled moving flat surface was developed, on which pairs of TLS scans were performed in various positions of the plane. In the stability evaluation process, the global test of the adjustment between the scan pairs was used. The results showed that the parameterization stability is conditioned to the positional relationship between the plane and the TLS at the moment of the scan.
\end{abstract}

Keywords: Terrestrial Laser Scanner. Parameterization. Global Test. 


\section{INTRODUÇÃO}

Nos últimos anos, as investigações quanto ao uso e a viabilidade da tecnologia laser scanner nos levantamentos geodésicos vem se intensificando. Uma das razões para isso está relacionada à possibilidade de adquirir densas nuvens de pontos à um curto espaço de tempo, ou seja, os avanços tecnológicos vêm permitindo que as varreduras com laser forneçam informações tridimensionais de superfícies de interesse a taxas de aquisição superiores a um milhão de pontos por segundo (WALTON; DELALOYE; DIEDERICHS, 2014). Isto fomenta as pesquisas de aplicação destes em trabalhos como monitoramento geodésico, reconstrução tridimensional de estruturas históricas, entre outros (GENECHTEN, 2008; PETRIE; TOTH, 2018).

No caso das aplicações ao monitoramento geodésico, as estruturas como túneis, pontes, barragens e torres de concreto são exemplos de objetos de estudos já investigadas a partir de levantamentos de varredura lasers, principalmente no tocante ao Laser Scanner Terrestre (LST) (SCHNEIDER, 2006; LOVAS et al., 2008; LENARTOVICZ, 2013; WALTON; DELALOYE; DIEDERICHS, 2014; GONÇALVES, 2018). Ressalta-se que a realização do monitoramento possibilita a mensuração e detecção de deslocamentos entre diferentes épocas, causados por fatores externos e internos, fazendo-se necessária a abundância de dados precisos sobre a estrutura.

A pluralidade de LST presente no mercado expõe a diversidade de composições possíveis nestes sistemas, fato que acarreta na dificuldade de classificação destes equipamentos (FRÖHLICH; METTENLEITER, 2004). Este fato fomenta a necessidade de investigações da aplicação destes instrumentos e a potencialidade dos produtos derivados das varreduras laser terrestre ao monitoramento, delineando as possíveis restrições oferecidas por estes sistemas e no impacto das incertezas contidas nos dados levantados.

Entretanto, uma das possibilidades de classificação de LST condiz com as abordagens de levantamentos disponíveis. De acordo com Petrie e Toth (2018), o modo de levantamento com LST pode ser distinguido entre o estático, em que o equipamento permanece parado ao longo do processo de varredura, e o levantamento dinâmico, no qual o sensor laser encontra-se em movimento a bordo de veículos terrestres, por exemplo. Em relação às técnicas de medição, Vosselman e Maas (2010) distinguem em time-of-flight (tempo de voo), Diferença de Fase ou Triangulação, sendo cada uma dessas técnicas diferenciadas não só pelos métodos de mensuração em si, mas quanto ao impacto nas precisões finais dos dados.

Como mencionado anteriormente, o LST visa a determinação de dados tridimensionais (pontos 3D), sendo estes vinculados a um sistema de referência local, elaborado em cada posição onde se realiza a varredura. Estes pontos são obtidos a partir de observações de ângulos verticais, ângulos horizontais (eixo de rotação do instrumento) e a medida da distância entre o equipamento e o alvo (VOSSELMAN; MAAS, 2010). Baseandose na impossibilidade de obtenção de dados com ausência de erros, sabe-se que uma das fontes de erro possível são os próprios instrumentos geodésicos, devido às imperfeições na elaboração e montagem dos equipamentos. Além destas imperfeições, erros provenientes da interação do laser com o meio de propagação e com a superfície refletora também são fatores que podem acrescer nas imprecisões das varreduras com LST.

De acordo com Holst e Kuhlmann (2016), além dos erros citados acima, a utilização do LST na investigação de deformações estruturais esbarra em outras dificuldades, como na ausência de pontos présinalizados e a não ocorrência de reamostragem sobre pontos de interesse no objeto levantado (entre épocas distintas de levantamento). Estes e outros fatores vêm resultando na busca pela parametrização da nuvem de pontos como uma alternativa de tratamento dos dados para fins de monitoramento. Todavia, este processo acarreta em alguns desafios, como na definição ideal da disposição da posição do LST e na interferência da quantidade de pontos necessária; na determinação do modelo de parametrização que possibilite a detecção de deformação desconhecidas; na consideração dos erros do modelo e das simplificações adotados sobre o objeto; na investigação dos métodos para correlacionar as observações com LST; e na influência dos erros sistemáticos do LST nas observações (HOLST; KUHLMANN, 2016).

Os elementos geométricos como planos, esferas e cilindros são alternativas de parametrização dos dados de varredura laser. A detecção de planos e determinação de parâmetros representativos destes é uma das abordagens utilizadas por pesquisadores nas atividades de monitoramento de estrutura. Alguns autores demonstram que o ajustamento das observações contidas no elemento plano fornece parâmetros com precisões melhores aos dados de varredura utilizados (LINDENBERGH; PFEIFER, 2005; BARBARELLA et al., 2017). 
Para Monserrat e Crosetto (2008) a principal vantagem deste método está na capacidade de detectar deslocamentos abaixo das precisões nominais, além da quantidade das observações adquiridas favorecer a alta redundância para a estimativa de parâmetros a serem calculados, baseando-se no método dos mínimos quadrados (MMQ). Sabe-se que a determinação de dados representativos sobre uma dada grandeza, a partir de múltiplas observações e suas inconsistências, é apenas viável por meio do ajustamento destes dados. Aplicando-se métodos que permitem a minimização das incertezas contidas nas observações é possível fazer com que as observações atendam condições desejadas (GHILANI, 2018).

Entretanto, apesar de alguns trabalhos de monitoramento estrutural apresentarem resultados satisfatórias quanto a utilização do ajustamento dos dados, derivados das varreduras com LST, em elementos geométricos (LINDENBERGH; PFEIFER, 2005; SCHNEIDER, 2006; JAAFAR; MENG; SOWTER, 2017; BARBARELLA et al., 2017), ainda cabem estudos em ambientes controlados que investiguem o uso, a estabilidade e a confiabilidade alcançável do processo de parametrização dos dados de varredura laser em planos, para fins de monitoramento geodésico. Simultaneamente, essa avaliação deve ponderar alguns desafios relacionados a parametrização, como a influência das posições das estações de varredura e do modelo matemático aplicável que contenha parâmetros eficientes para a deteç̧ão dos deslocamentos desconhecidos (HOLST; KUHLMANN, 2016).

Com o objetivo de investigar a confiabilidade da abordagem de utilização de elementos planos no monitoramento geodésico de estruturas, neste artigo propõem-se a realização de uma avaliação laboratorial da estabilidade da parametrização de dados tridimensionais de um plano de controle levantado a partir das varreduras com LST.

\section{MATERIAL E MÉTODOS}

Para a realização da avaliação proposta, elaborou-se um protótipo contendo uma superfície plana apresentado na Figura 1, denominada Plano de Controle Experimental (PCE). Sobre esta superfície foram efetuados pares de varredura com o LST, relativos a cada posição do PCE, mantendo-se a mesma posição do instrumento. Posteriormente, os parâmetros dos planos foram calculados e submetidos a avaliações estatísticas para determinação da igualdade entre os pares de varredura, ou seja, da semelhança entre os planos ajustados.

Figura 1 - Protótipo de plano de controle experimental.

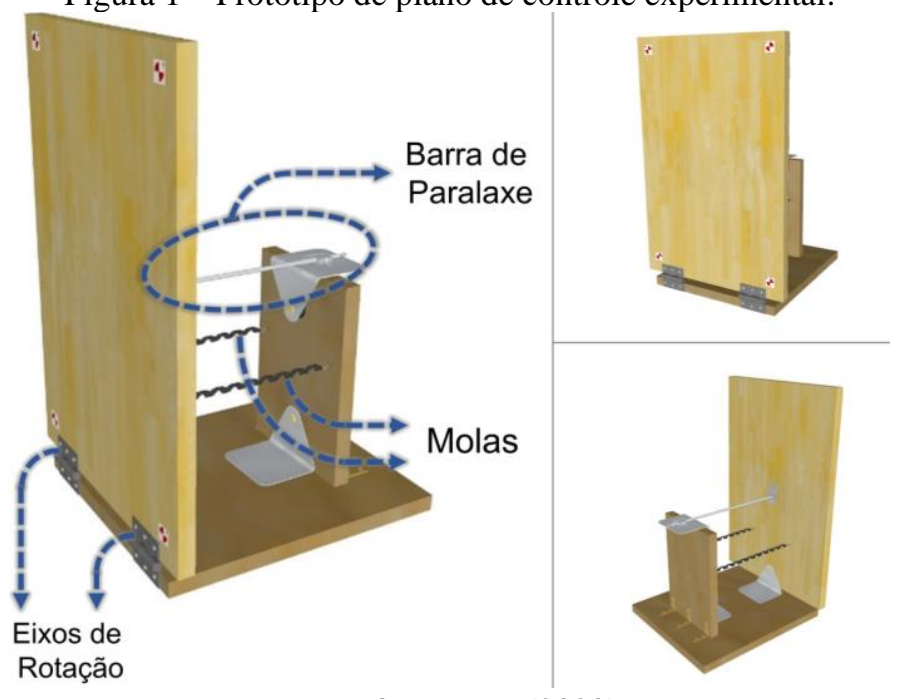

Fonte: Os autores (2020).

\subsection{Laser Scanner Terrestre BLK360}

O LST BLK360, desenvolvido pela empresa Leica Geosystems, possui uma unidade laser com 830 $\mathrm{nm}$ comprimento de onda. O prisma rotativo vertical e a base com giro horizontal permitem a realização do levantamento laser do tipo panorâmico. Além destes, o sistema é integrado com três câmeras HDR de 150 megapixels, as quais viabilizando a obtenção de imagens esféricas $\left(360^{\circ}\right)$ e a determinação de cores como 
elementos adicionais para cada ponto mensurado (LEICA GEOSYSTEMS, 2018).

Ao longo do levantamento, a taxa de aquisição dos pontos pode chegar a 360 mil por segundo. $\mathrm{O}$ alcance de varredura mínimo é de $60 \mathrm{~cm}$ de distância, a partir da posição do instrumento, e máximo de $60 \mathrm{~m}$. A precisão nominal dos pontos tridimensionais, para o sistema laser, é de $4 \mathrm{~mm}$ a $10 \mathrm{~m} \mathrm{e} 7 \mathrm{~mm}$ a $20 \mathrm{~m}$ (LEICA GEOSYSTEMS, 2018).

O laser BLK360 possibilita a realização do escaneamento em três modos: Rápido, Padrão e Alta densidade. A configuração altera diretamente o tempo de duração das varreduras, variado de 0,67 min à 3,67 min para o modo de alta densidade (LEICA GEOSYSTEMS, 2018).

Na Figura 2 são apresentados os campos de varredura panorâmica vertical (a) e horizontal (b) de registro dos pontos, com varredura do laser de $300^{\circ}$ e $360^{\circ}$, respectivamente. Cabe ressaltar que o BLK360 apenas realiza varreduras no modo panorâmico.

Figura 2 - Campos de varredura do laser scanner terrestre BLK360.

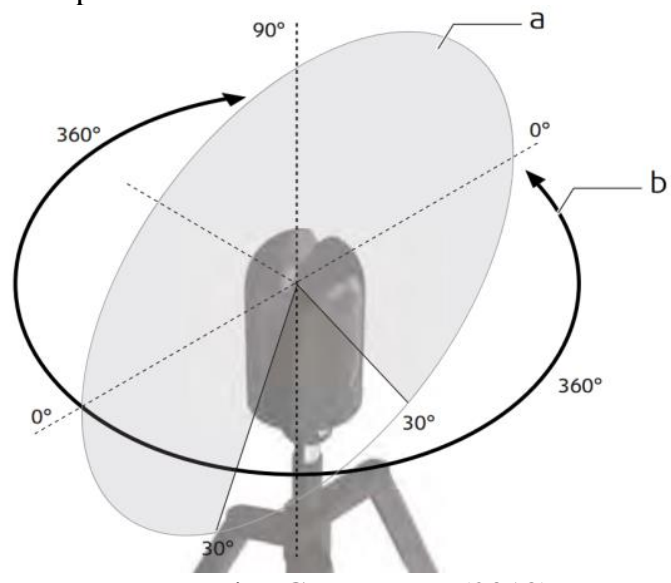

Fonte: Leica Geosystems (2018).

A comunicação do LST com equipamentos externos (Tablet e dispositivo de computação) segue a tendência atual de comunicação por conexão sem fio, utilizando a tecnologia Wi-Fi a partir de uma antena WLAN $360^{\circ}$ presente na base do instrumento.

\subsection{Plano de Controle Experimental (PCE)}

O PCE é constituído por uma placa de madeira fixada à uma base horizontal por meio de duas dobradiças metálicas. Estas peças viabilizam a movimentação do plano com uma rotação $\theta$, impulsionado por uma barra de paralaxe adaptada à posterior da placa. A barra possui uma gradação submilimétrica, permitindo a quantificação da movimentação da placa no sentido da barra. O condicionamento da movimentação do plano no PCE, proporcional ao deslocamento da barra, foi viabilizado por meio da fixação de molas na face posterior do plano estabelecido (Erro! Fonte de referência não encontrada.2).

A barra foi fixada paralelamente à base do sistema e encontra-se perpendicular ao plano quando este também está perpendicular à base do protótipo (posição inicial). Para determinar o ângulo de rotação $\theta$, com base na variação de comprimento da barra, necessitou-se a mensuração da altura entre o ponto de incidência da barra de paralaxe e a base do protótipo (centro de rotação da dobradiça). Com a Eq. (1) calcula-se o ângulo $\theta$ a partir da h da barra de paralaxe em relação a base do protótipo e os deslocamentos 1 da barra de paralaxe.

$$
\operatorname{tg} \theta=\frac{l}{h}
$$

Determinou-se a altura entre o eixo de rotação da placa e o ponto de incidência da barra de paralaxe, medida em 0,3279 m. Conforme a Figura 3, esta determinação foi realizada empregando-se nivelamento geométrico de precisão com o nível DNA03 da Leica Geosystems, no qual a mira foi posicionada na base do PCE e sobre a barra da hasta de paralaxe, descontando-se a distância entre a base do protótipo e o eixo de rotação da 
dobradiça e a diferença entre a parte superior da barra de paralaxe (onde a mira foi apoiada) e do ponto de incidência no plano, sendo estas duas grandezas medidas com um paquímetro.

Figura 3 - Determinação da altura do ponto de incidência da barra de paralaxe.

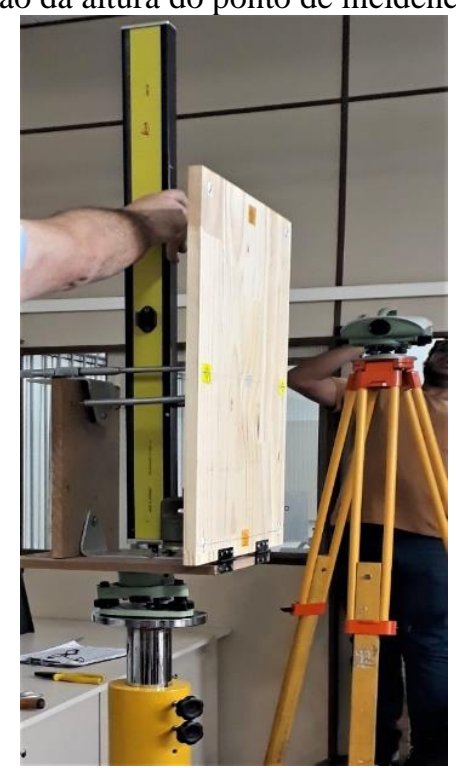

Fonte: Os autores (2020).

Visto que neste trabalho é proposto uma avaliação estatística para o monitoramento de estruturas, as variações aplicadas na barra de paralaxe foram inferiores, próximas e superiores as grandezas de 10 e 15 milímetros. Estes valores foram adotados por Jerke (2019) como as variações máxima tolerável, horizontal e vertical respectivamente, em que se analisou o monitoramento tridimensional com alvos a jusante de uma barragem.

\subsection{Aquisição das observações}

A precisão das medições por varredura laser depende das condições do levantamento, como a configuração do escaneamento (posição do scanner e objeto levantado), a geometria da superfície do objeto e a refletividade do objeto (SCHMITZ et al., 2019).

As varreduras com LST foram realizados em duas etapas, modificando-se a posição relativa entre o instrumento e o PCE em cada uma delas. Conforme ilustrado no fluxograma a baixo (Figura 4), na $1^{\text {a }}$ etapa o LST foi estacionado em posição frontal ao PCE, visando o levantamento de coordenadas tridimensionais com os ângulos de incidência, formado entre o feixe laser e a superfície, próximos à $90^{\circ}$ (Figura 5-a). Na $2^{\mathrm{a}}$ etapa, o instrumento foi movido para posição oblíqua ao PCE e novas varreduras foram feitas, com ângulos de incidência próximos à 45 (Figura 5-b). 
Figura 4 - Fluxograma das etapas de aquisição dos dados.

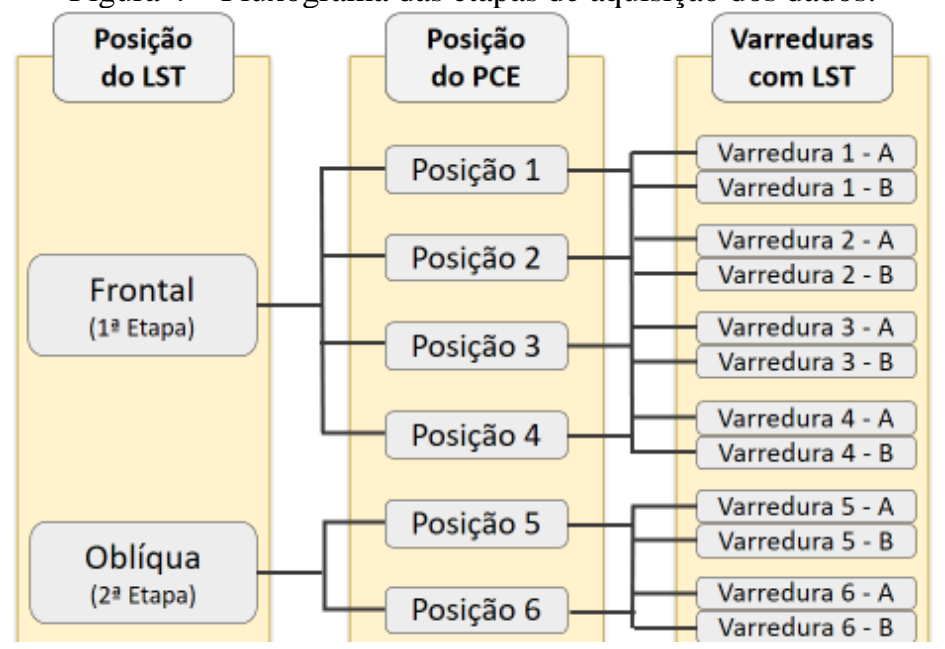

Fonte: Os autores (2020).

Figura 5 - Posições de LST nas etapas de levantamento.

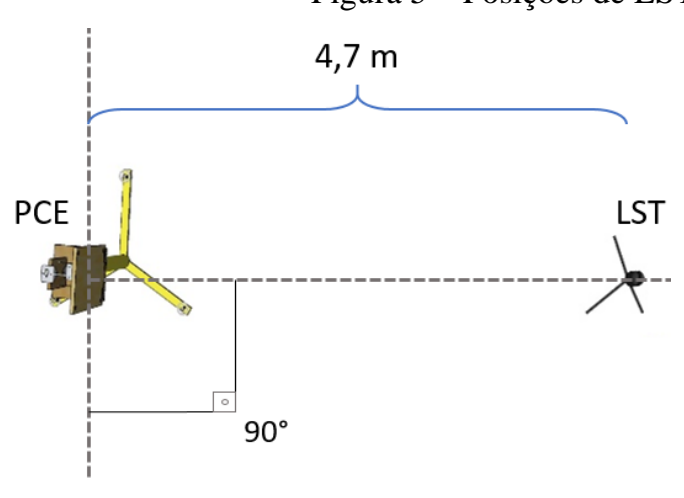

(a)

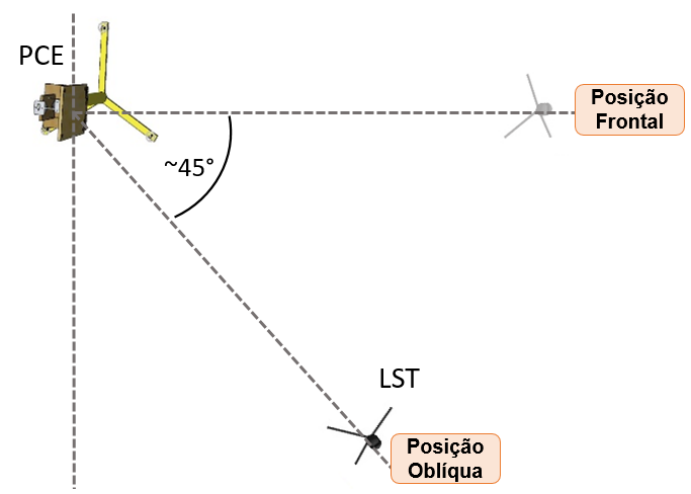

(b)

Fonte: Os autores (2020).

A Figura 6 ilustra os deslocamentos aplicados sobre o plano, resultando nas seis posições avaliadas. Nesta, o plano $\pi_{0}$ inicial sofre um deslocamento por meio de uma rotação, tendo como eixo sua base, resultado nas posições indicadas como $\pi_{1}$ e $\pi_{2}$. O resultado dos deslocamentos são as inclinações demonstradas como $\theta_{1}$ e $\theta_{2}$.

Figura 6 - Deslocamentos e inclinação do plano.

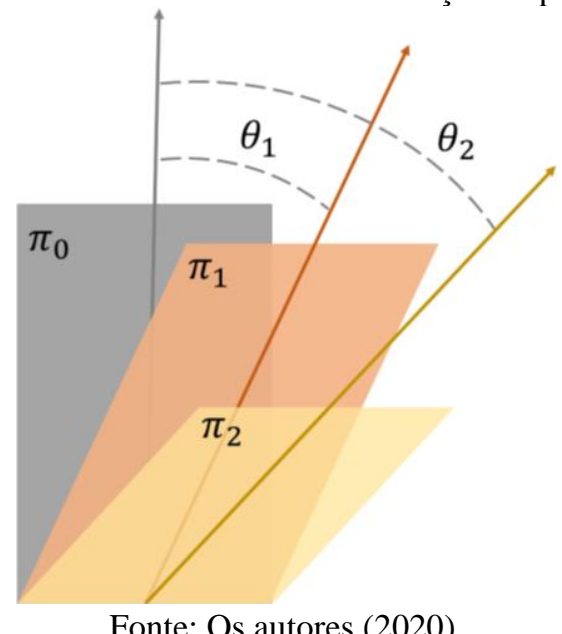

A distância entre o LST e o PCE foi de 4,7 m, mantendo-se o LST estacionado na mesma posição em todas as varreduras. Assim, evitou-se que outros processos fossem realizados, como o georreferenciamento ou alinhamento dos dados, uma vez que estas técnicas podem propagar erros aos dados, afetando a confiabilidade 
na parametrização (VOSSELMAN; MAAS, 2010).

As varreduras foram importadas individualmente no software Cyclone Register e realizada a segmentação manual das nuvens de pontos brutas para seleção apenas dos pontos levantados sobre o plano, visto que o BLK360 realiza, apenas, levantamento no modo panorâmico. Pontos sobre as dobradiças fixadas no plano também foram excluídos.

As nuvens segmentadas foram exportadas no formato *.PTS, possibilitando a utilização dos dados em outras plataformas. Em seguida, os arquivos foram salvos no formato *.TXT para utilização dos mesmo para o ajustamento no software MATLAB versão 2017.

\subsection{Parametrização}

Para avaliar a estabilidade da parametrização dos dados, propôs-se trabalhar com o elemento plano. Conforme Venture (2015), um plano pode ser define a partir da Eq. (2), denominada equação fundamental do plano.

$$
a x+b y+c z=d
$$

onde $\mathrm{x}$, y e z representam as coordenadas dos pontos pertencentes ao plano; a, b e c são parâmetros definidores do plano e configuram o vetor normal do plano; d não define o plano (ERDÉLYI, 2017), mas representa um valor constante a expressão $a x+b y+c z$ para todo ponto $\mathrm{P}=(\mathrm{x}, \mathrm{y}, \mathrm{z})($ LIMA, 2014).

De acordo com Boulos e Camargo (1987), os planos $\pi_{1}: a_{1} x+b_{1} y+c_{1} z+d=0$ e $\pi_{2}: a_{2} x+b_{2} y+c_{2} z$ $+d=0$ são semelhantes ou coincidentes se, para a mesma solução (x, y, z) (LIMA, 2014), existir um valor constante $\lambda \neq 0$ tal que (Eq. 3, 4, 5 e 6):

$$
\begin{aligned}
a_{1} & =\lambda a_{2} \\
b_{1} & =\lambda b_{2} \\
c_{1} & =\lambda c_{2} \\
d_{1} & =\lambda d_{2}
\end{aligned}
$$

Como ilustrado pela Figura 7, $\pi_{1}$ e $\pi_{2}$ são coincidentes quando os vetores $\left(a_{1}, b_{1}, c_{1}\right)$ e $\left(a_{2}, b_{2}, c_{2}\right)$ são múltiplos (Figura 7-a). Por outro lado, nos casos $\pi_{1}$ e $\pi_{2}$ em que são concorrentes (Figura 7-b), haverá interseção entre ambos (reta r) e $\left(a_{1}, b_{1}, c_{1}\right)$ e $\left(a_{2}, b_{2}, c_{2}\right)$ não são múltiplos.

Figura 7 - Planos coincidentes e concorrentes.

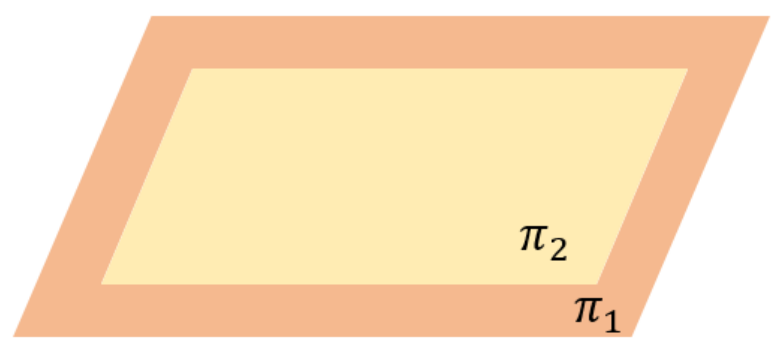

(a)

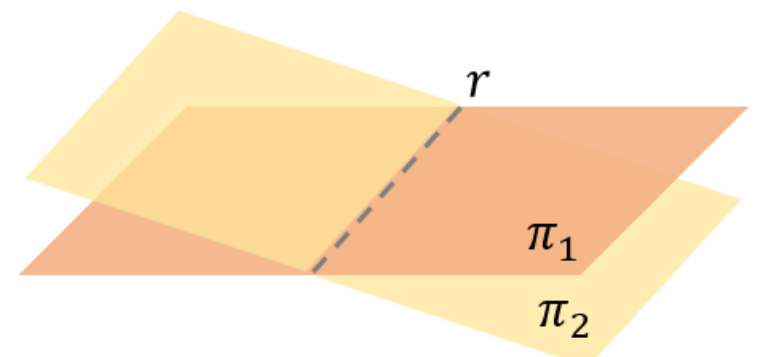

(b)

Fonte: Os autores (2020).

Neste trabalho, a determinação destes parâmetros foi realizada por meio do ajustamento das observações utilizando o método combinado. Conforme a equação do plano demonstrada acima, cada equação relaciona os parâmetros definidores do plano $a, b$ e c e três observações cartesianas dos pontos contidos nele x, y e z. Portanto, cabe a adoção de um ajustamento pelo método combinado, uma vez que, para o modelo paramétrico cada equação deveria estar ligada a uma única grandeza mensurável. Sendo assim, optou-se pela realização do ajustamento simultâneo entre os parâmetros e as observações, conforme a Eq. (7) (GEMAEL; 
MACHADO; WANDRESEN, 2015):

$$
F\left(L_{a}, X_{a}\right)=0
$$

em que $L_{a}$ representa o vetor dos valores observados ajustados e $X_{a}$ o vetor dos parâmetros ajustados.

Considerando $L_{a}=L_{b}+V$ e $X_{a}=X_{0}+X$, onde $L_{b}$ é o vetor dos valores observados, $V$ o vetor dos resíduos, $X_{0}$ o vetor dos parâmetros iniciais estimados e $X$ o vetor correção dos parâmetros, obtém-se a Eq. (8). Em seguida, a linearização da expressão por Série de Taylor, em relação ao vetor das observações e ao vetor dos parâmetros ajustados, resulta na aproximação apresentada na Eq. (12) (GEMAEL; MACHADO; WANDRESEN, 2015):

$$
\begin{gathered}
F\left(L_{a}, X_{a}\right)=F\left(L_{b}+V, X_{0}+X\right) \\
F\left(X_{0}+X, L_{b}+V\right) \cong F\left(X_{0}, L_{b}\right)+\frac{\partial F}{\partial X_{a}}\left(X_{a}-X_{0}\right)+\frac{\partial F}{\partial L_{a}}\left(L_{a}-L_{b}\right)=0 \\
A=\frac{\partial F}{\partial X_{a}}=\left[\begin{array}{ccc}
\frac{\partial F_{1}}{\partial a} & \frac{\partial F_{1}}{\partial b} & \frac{\partial F_{1}}{\partial c} \\
\frac{\partial F_{2}}{\partial a} & \frac{\partial F_{2}}{\partial b} & \frac{\partial F_{2}}{\partial c} \\
\frac{\partial F_{3}}{\partial a} & \frac{\partial F_{3}}{\partial b} & \frac{\partial F_{3}}{\partial c} \\
\vdots & \vdots & \vdots \\
\frac{\partial F_{n}}{\partial a} & \frac{\partial F_{n}}{\partial b} & \frac{\partial F_{n}}{\partial c}
\end{array}\right] \\
B=\frac{\partial F}{\partial L_{a}}=\left[\begin{array}{ccc}
\frac{\partial F_{1}}{\partial x_{1}} & \frac{\partial F_{1}}{\partial y_{1}} & \frac{\partial F_{1}}{\partial z_{1}} \\
\frac{\partial F_{2}}{\partial x_{2}} & \frac{\partial F_{2}}{\partial y_{2}} & \frac{\partial F_{2}}{\partial z_{2}} \\
\frac{\partial F_{3}}{\partial x_{3}} & \frac{\partial F_{3}}{\partial y_{3}} & \frac{\partial F_{3}}{\partial z_{3}} \\
\vdots & \vdots & \vdots \\
\frac{\partial F_{n}}{\partial x_{n}} & \frac{\partial F_{n}}{\partial y_{n}} & \frac{\partial F_{n}}{\partial z_{n}}
\end{array}\right] \\
A X+B V+W=0
\end{gathered}
$$

onde a matriz $A$ consiste na matriz das derivadas parciais da equação de $n$ condição em relação aos u parâmetros (a, b, c), a matriz $B$ nas derivadas das equações de condições em relação as $n$ observações e $F\left(L_{b}, X_{0}\right)=W$ o vetor erro de fechamento, ou seja, valores calculados a partir das $r$ equações de condição considerando as observações realizadas (contendo erros) e os valores iniciais estimados para parâmetros (GEMAEL; MACHADO; WANDRESEN, 2015).

A resolução das equações normais pode ser representada pelo sistema apresentado a seguir, contendo hipermatrizes (matrizes cujos elementos são matrizes) (GEMAEL; MACHADO; WANDRESEN, 2015):

$$
\left[\begin{array}{l}
V \\
K \\
X
\end{array}\right]=-\left[\begin{array}{ccc}
P & -B^{T} & 0 \\
B & 0 & A \\
0 & A & 0
\end{array}\right]^{-1}\left[\begin{array}{c}
0 \\
W \\
0
\end{array}\right]
$$

Desenvolvendo o sistema acima, tem-se:

$$
\begin{gathered}
V=P^{-1} B^{T} K \\
K=-M^{-1}(A X+W) \\
X=-\left(A^{T} M^{-1} A\right)^{-1} A^{T} M^{-1} W
\end{gathered}
$$


em que $M$ representa o produto matricial dado por $M=B P^{-1} B^{T}$ e $P$ designa a matriz peso do ajustamento.

A matriz peso do ajustamento foi elaborada com base na precisão nominal do instrumento e a distância entre o LST e o plano de controle experimental. A partir das precisões nominais $4 \mathrm{~mm}$ a $10 \mathrm{~m}$ e $7 \mathrm{~mm}$ a $20 \mathrm{~m}$ indicadas pelo fabricante, definiu-se a equação reduzida da reta, para as duas precisões, por Regressão Linear no software Excel. Assim, foi elaborada a Eq. (17) para determinação da qualidade dos pontos, de acordo com a distância entre instrumento e a superfície:

$$
D P=(0,0003 * \rho)+0,001
$$

onde $D P$ é o desvio-padrão do ponto levantado e $\rho$ a distância entre o instrumento e a superfície levantada.

Com base na Eq. (17) e na distância de 4,7 m entre o LST e o plano de controle, o desvio-padrão calculado, com base na precisão nominal, foi de $0,00241 \mathrm{~m}(2,41 \mathrm{~mm})$. Considerando que este valor estimado corresponde a precisão 3D do ponto e que o ajustamento é aplicado sobre as coordenadas x, y e z, o inverso da variância (variância $=D P^{2}$ ) foi dividido por 3, compondo a matriz diagonal da matriz-peso do ajustamento.

A partir da Eq. (16) é possível determinar o vetor dos parâmetros ajustados (GEMAEL; MACHADO; WANDRESEN, 2015). Neste trabalho, o vetor dos parâmetros estimados foi dado por $X_{0}=[1,1,1]$, enquanto o elemento $d$ estabelecido com o valor constante 1 para o ajustamento de todos os planos levantados.

Para o cálculo das respectivas precisões dos parâmetros ajustados $\left(\Sigma_{X_{a}}\right)$, determina-se a matriz variância-covariância $(M V C)$ dos mesmos a partir da lei de propagação na Eq. (18), em que são propagadas as variâncias e covariâncias das observações sobre os parâmetros ajustados (GEMAEL; MACHADO; WANDRESEN, 2015):

$$
\Sigma_{X_{a}}=\hat{\sigma}_{0}^{2}\left(A^{T} M^{-1} A\right)^{-1}
$$

onde $\hat{\sigma}_{0}^{2}$ representa a variância da observação de peso unitário, determinada por meio da relação $\hat{\sigma}_{0}^{2}=\frac{V^{T} P V}{g l}$ (GEMAEL; MACHADO; WANDRESEN, 2015), em que $g l$ corresponde aos graus de liberdade.

\subsection{Análise de estabilidade dos parâmetros}

O teste de estabilidade se baseou na comparação entre pares de varreduras com LST (planos homólogos) realizadas para 6 posições distintas do PCE, conforme apresentado no Quadro 1 abaixo:

Quadro 1 - Testes realizados para detecção de deslocamento entre planos.

\begin{tabular}{|l|l|}
\hline \multicolumn{2}{|c|}{ Posição Frontal } \\
\hline Teste 1 & Varredura 1-A x Varredura 1-B \\
\hline Teste 2 & Varredura 2-A x Varredura 2-B \\
\hline Teste 3 & Varredura 3-A x Varredura 3-B \\
\hline Teste 4 & Varredura 4-A x Varredura 4-B \\
\hline \multicolumn{2}{|c|}{ Posição Oblíqua } \\
\hline Teste 5 & Varredura 5-A x Varredura 5-B \\
\hline Teste 6 & Varredura 6-A x Varredura 6-B \\
\hline
\end{tabular}

Fonte: Os autores (2020).

O teste global do ajustamento é uma metodologia usualmente utilizada na detecção de erros não aleatórios nas observações, assim como o procedimento Baarda's Data Snooping para detecção e identificação de outliers (KLEIN, 2011; ROFATTO et al., 2018). Aydin (2012) apresentou uma abordagem para detecção de deslocamentos em redes geodésicas baseada na avaliação pelo teste global.

De acordo com Aydin (2012), o deslocamento $\Delta$ entre duas observações $\kappa_{l}$ e $\kappa_{2}$ realizadas sobre uma mesma grandeza, em épocas distintas (Eq. (19)), pode ser detectado a partir da rejeição da hipótese nula, com um nível $\alpha$ de significância e considerando a soma das matrizes variância-covariância da época $1\left(\Sigma_{\kappa l}\right)$ e época $2\left(\Sigma_{\kappa 2}\right)$. Aceitando-se a hipótese nula, indica-se a não ocorrência de deslocamento entre as observações, 
conforme demonstrado a seguir:

$$
\begin{gathered}
\Delta=\kappa_{2}-\kappa_{1} \\
\Sigma_{\Delta}=\Sigma_{\kappa 1}+\Sigma_{\kappa 2} \\
H_{0}: E(\Delta)=0 \\
H_{1}: E(\Delta) \neq 0
\end{gathered}
$$

Sendo assim, a estabilidade dos planos homólogos foi investigada por meio das possíveis discrepâncias calculadas entre os parâmetros a, b e c dos pares testados. Sendo assim, os vetores deslocamento $\Delta_{t o t}$ foram obtidos conforme a Eq. (23):

$$
\Delta_{t_{0} t}=\left[\begin{array}{l}
a_{t}-a_{t_{0}} \\
b_{t}-b_{t_{0}} \\
c_{t}-c_{t_{0}}
\end{array}\right]
$$

onde $a_{t}, b_{t}$ e $c_{t}$ representam os parâmetros dos planos sobre os quais se investiga a estabilidade, na época $t$, enquanto $a_{t 0}, b_{t 0}$ e $c_{t 0}$ configuram os parâmetros do plano com a posição de referência, na época $t_{0}$.

A $M V C$ a ser aplicada no teste é a resultante da soma dos elementos $\sigma_{i j}$ da $M V C$ obtida pelo ajustamento dos dados na época $t_{0}\left(\Sigma_{t 0}\right)$ e na época $t\left(\Sigma_{t}\right)$. Portanto, cada $M V C$ a ser utilizada no somatório corresponde as variâncias e covariâncias dos parâmetros ajustados a, b e c que representam o plano em cada varredura realizada, como apresentado na Eq. (24).

$$
\Sigma_{t_{0}}+\Sigma_{t}=\Sigma_{\Delta_{t_{0} t}}=\left[\begin{array}{ccc}
\sigma_{a_{t_{0}}}^{2}+\sigma_{a_{t}}^{2} & \sigma_{a_{t_{0}} b_{t_{0}}}+\sigma_{a_{t} b_{t}} & \sigma_{a_{t_{0}} c_{t_{0}}}+\sigma_{a_{t} c_{t}} \\
\sigma_{a_{t_{0}} b_{t_{0}}}+\sigma_{a_{t} b_{t}} & \sigma_{b_{t_{0}}}^{2}+\sigma_{b_{t}}^{2} & \sigma_{b_{t_{0}} c_{t_{0}}}+\sigma_{b_{t} c_{t}} \\
\sigma_{a_{t_{0}} c_{t_{0}}}+\sigma_{a_{t} c_{t}} & \sigma_{b_{t_{0}} c_{t_{0}}}+\sigma_{b_{t} c_{t}} & \sigma_{c_{t_{0}}}^{2}+\sigma_{c_{t}}^{2}
\end{array}\right]
$$

Klein (2011) enfatiza que o teste de hipótese mais adequado para detecção de erros é o unilateral. De acordo com Aydin (2012), o teste global pode ser realizado com duas abordagens: Pela distribuição F ou pela distribuição Qui-quadrado. O autor indica o teste F como o mais aplicado em análises de deformações e o teste Qui-quadrado como a alternativa aplicada quando o sigma a priori é precisamente conhecido. Entretanto, o teste Qui-quadrado é apontado como o mais robusto, o que significa que maiores magnitudes de deslocamento ou redes mais precisas seriam necessárias para que a avaliação pelo teste F atingisse a mesma potência do Quiquadrado.

Cabe ressaltar que um teste de hipótese, como o aplicado neste método, apenas indica a existência ou não de possíveis deslocamentos nas observações (KLEIN, 2011). Logo, para análises de deslocamentos, o teste global tem como objetivo a indicar a estabilidade entre as grandezas avaliadas fundamentado em um nível de confiança estabelecido, e não a magnitude dos deslocamentos entre elas.

A Eq. (25) apresenta o valor da estatística calculada $\left(X_{\text {calculado }}^{2}\right)$ a partir da diferença entre os parâmetros, da $M V C$ resultante e do sigma a-priori $\left(\sigma_{0}^{2}\right)$, o qual, neste trabalho, foi arbitrado em 1 . Este valor foi comparado com a estatística tabelada $\left(X_{\text {teórico(gl, }, \mathrm{a})}^{2}\right.$ ), determinada pelos graus de liberdade da amostra e pelo nível de significância $(\alpha)$.

$$
X_{\text {calculado }}^{2}=\frac{\Delta^{T} \Sigma_{\Delta}^{-1} \Delta}{\sigma_{0}^{2}}
$$

Conforme Aydin (2012), para as avaliações em que se utiliza o teste Qui-quadrado, os graus de liberdade são condizentes às dimensões da $M V C$ aplicada. Neste estudo, de acordo com a Eq. (24) apresentada, as dimensões estão relacionadas ao número de parâmetros testado $(u=3)$. Sendo assim, para os testes de semelhança entre os planos, utilizou-se $g l=u=3$, uma vez que a $M V C$ possui dimensão 3 x 3 . O nível de significância foi estabelecido em $5 \%(\alpha=0,05)$. 
Se a relação $X_{\text {calculado }}^{2} \leq X_{\text {teórico(gl,a) }}^{2}$ for atendida, então a hipótese nula é aceita, indicando que não há deslocamento entre os parâmetros testados, a um nível de significância $\alpha$ e com u graus de liberdade. Mas se $X_{\text {calculado }}^{2}>X_{\text {teórico(gl,a) }}^{2}$, logo, rejeita-se a hipótese nula, indicando a não estabilidade na determinação dos planos.

\section{RESULTADOS E DISCUSSÃO}

O experimento foi realizado com base nos dados obtidos a partir das varreduras com LST sobre o PCE. Neste trabalho, buscou-se avaliar a influência da variação da posição do plano em relação ao sistema de referência local do laser. Assim, a Tabela 1 apresenta as 6 posições distintas do PCE com base nos deslocamentos lineares e angulares.

Tabela 1 - Quantificação dos deslocamentos do plano de controle experimental.

\begin{tabular}{l|c|c|c}
\hline \multicolumn{1}{c|}{ Posição do LST } & Varreduras & $\begin{array}{c}\text { Deslocamento da Barra de } \\
\text { Paralaxe (mm) }\end{array}$ & Rotação do plano $\theta$ \\
\hline \multirow{3}{*}{ Frontal } & 1-A e 1-B & 0,000 & $0^{\circ}$ \\
& 2-A e 2-B & 5,160 & $0^{\circ} 53^{\prime}$ \\
& 3-A e 3-B & 9,180 & $1^{\circ} 35^{\prime}$ \\
\multirow{2}{*}{ Oblíquo } & 4-A e 4-B & 20,150 & $3^{\circ} 28^{\prime}$ \\
\hline
\end{tabular}

Fonte: Os autores (2020).

Para evitar que a variação dos fatores ambientais interferisse na avaliação metodológica, os testes foram realizados em laboratório, o que possibilitou a verificação da temperatura, as quais ficaram entre 18,8 ${ }^{\circ} \mathrm{C}$ e $19,3{ }^{\circ} \mathrm{C}$.

Para cada posição acima, duas varreduras foram realizadas, totalizando 12 planos levantados. Seguindo o tratamento dos dados, o ajustamento das observações foi realizado pelo método combinado, adotando-se a Eq. (2) citada anteriormente como o modelo funcional. Assim, foram determinados os parâmetros a, b e c de cada plano e, simultaneamente, os respectivos desvios-padrão $\sigma a, \sigma b$ e $\sigma c$ para de cada parâmetro, conforme apresentado na Tabela 2.

Tabela 2 - Parâmetros ajustados dos planos.

\begin{tabular}{c|c|c|c|c|c|c|c|c}
\hline $\begin{array}{c}\text { Posição do } \\
\text { LST }\end{array}$ & Varreduras & $\begin{array}{c}\mathbf{N}^{\mathbf{0}} \mathbf{d e} \\
\mathbf{p o n t o s}\end{array}$ & $\mathbf{a}$ & $\mathbf{\pm \sigma a}$ & $\mathbf{b}$ & $\mathbf{\pm \sigma b}$ & $\mathbf{c}$ & $\mathbf{\boldsymbol { \sigma c }}$ \\
\hline \multirow{5}{*}{ Frontal } & 1-A & 7481 & $-0,04132$ & 0,00010 & $-0,03368$ & 0,00010 & $-0,00021$ & 0,00009 \\
& 1-B & 7557 & $-0,04128$ & 0,00010 & $-0,03373$ & 0,00010 & $-0,00021$ & 0,00009 \\
& 2-A & 7481 & $-0,04128$ & 0,00010 & $-0,03371$ & 0,00010 & $-0,00098$ & 0,00009 \\
& 2-B & 7549 & $-0,04126$ & 0,00010 & $-0,03374$ & 0,00010 & $-0,00100$ & 0,00009 \\
& 3-A & 7515 & $-0,04126$ & 0,00010 & $-0,03372$ & 0,00010 & $-0,00165$ & 0,00009 \\
& 3-B & 7646 & $-0,04124$ & 0,00010 & $-0,03374$ & 0,00010 & $-0,00166$ & 0,00009 \\
& 4-A & 7615 & $-0,04119$ & 0,00010 & $-0,03374$ & 0,00010 & $-0,00339$ & 0,00009 \\
& 4-B & 7612 & $-0,04121$ & 0,00010 & $-0,03372$ & 0,00010 & $-0,00341$ & 0,00009 \\
\hline \multirow{5}{*}{ Oblíquo } & 5-A & 10400 & $-0,04818$ & 0,00011 & $-0,03689$ & 0,00014 & $-0,00001$ & 0,00009 \\
& 5-B & 10511 & $-0,04810$ & 0,00011 & $-0,03698$ & 0,00014 & $-0,00001$ & 0,00009 \\
& 6-A & 10725 & $-0,04814$ & 0,00011 & $-0,03689$ & 0,00014 & $-0,00147$ & 0,00009 \\
& 6-B & 7481 & $-0,04132$ & 0,00010 & $-0,03368$ & 0,00010 & $-0,00021$ & 0,00009 \\
\hline
\end{tabular}

Fonte: Os autores (2020).

Pode-se observar similaridades entre os parâmetros e os desvios-padrão em cada par de varredura, indicando uma estabilidade prévia nas parametrizações. Os maiores desvios-padrão foram observados nas varreduras 5-A, 5-B, 6-A e 6-B, indicando que quanto mais afastado de $90^{\circ}$ encontra-se o ângulo de incidência do feixe laser sobre a superfície levantada, pior é a precisão dos parâmetros determinados.

Para avaliar a semelhança entre os planos, calcularam-se as constantes que estabelece a relação entre os parâmetros dos pares homólogos de varredura, conforme a Tabela 3. 
Tabela 3 - Constantes de proporcionalidade entre os parâmetros dos planos.

\begin{tabular}{l|c|c|c}
\hline \multicolumn{1}{c|}{ Texto } & $\mathbf{a}_{\mathbf{A}} / \mathbf{a}_{\mathbf{B}}$ & $\mathbf{b}_{\mathbf{A}} / \mathbf{b}_{\mathbf{B}}$ & $\mathbf{c}_{\mathbf{A}} / \mathbf{c}_{\mathbf{B}}$ \\
\hline Teste 1 & 1,001 & 0,999 & 1,000 \\
Teste 2 & 1,000 & 0,999 & 0,980 \\
Teste 3 & 1,000 & 0,999 & 0,994 \\
Teste 4 & 1,000 & 1,001 & 0,994 \\
Teste 5 & 1,002 & 0,998 & 1,000 \\
Teste 6 & 1,001 & 0,999 & 0,993 \\
\hline
\end{tabular}

Fonte: Os autores (2020).

As relações determinadas a cima demonstram que a maioria dos planos ajustados podem ser considerados semelhantes, com constantes próximas a 1. A relação entre os parâmetros da Varredura 2- $A$ e 2$B$ apresentou o valor mais distante da constante 1 para os parâmetros $c_{A}$ e $c_{B}$, indicando que a variação do ângulo vertical pode interferir na parametrização do plano. O Teste 6 apresentou relações diferentes de 1 para os três parâmetros testados, reforçando a influência tanto da inclinação do plano, quanto o efeito negativo dos ângulos de incidência horizontais e verticais na parametrização dos levantamentos oblíquos.

Seguindo a avaliação proposta, a estabilidade na determinação dos parâmetros foi calculada a partir

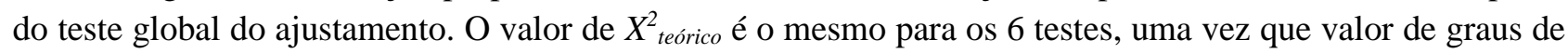
liberdade não se altera. Assim, para o nível de significância de 5\% e com três graus de liberdade, o valor crítico na distribuição $X^{2}$ é de 7,815 .

A Tabela 4 apresenta as estatísticas de teste calculadas para cada comparação e a detecção da estabilidade ou não entre as parametrizações dos planos homólogos.

Tabela 4 - Determinação das estatísticas de teste.

\begin{tabular}{l|c|c}
\hline \multicolumn{1}{c|}{ Teste de deslocamento } & $\mathbf{X}_{\text {calculado }}^{\mathbf{2}}$ & Estabilidade dos parâmetros \\
\hline Teste 1 & 0,5858 & $\mathrm{Sim}$ \\
Teste 2 & 1,0976 & $\mathrm{Sim}$ \\
Teste 3 & 0,000 & $\mathrm{Sim}$ \\
Teste 4 & 0,6266 & $\mathrm{Sim}$ \\
Teste 5 & 0,5372 & $\mathrm{Sim}$ \\
Teste 6 & 2,3437 & $\mathrm{Sim}$ \\
\hline
\end{tabular}

Fonte: Os autores (2020).

Com base nos valores das estatísticas a cima, a hipótese nula do não deslocamento entre os planos homólogos foi aceita, com nível de significância de $95 \%$ e com 3 graus de liberdade. $O$ valor do $X_{\text {calculado }}^{2}$ mais discrepante foi determinado para o Teste 6 , indicando que dentre os pares de varreduras testados, a Varredura 6- $A$ e 6-B apresentaram maiores chances de não semelhança entre os planos. Este fato reforça a ideia de que a inclinação da superfície varrida se torna mais sensível a interferências para a parametrização em plano para o caso das varreduras oblíquas.

\section{CONCLUSÃO}

A investigação em laboratório demonstrou que a utilização dos parâmetros, determinados a partir de nuvens de pontos, torna-se uma alternativa viável pela confiabilidade alcançável dos dados, com precisões melhores às estimadas aos pontos ajustados. Sendo assim, confirma-se a possibilidade de uso dos parâmetros do elemento plano em processos como, por exemplo, no registro de nuvens de pontos e no monitoramento de estruturas.

Entretanto, os resultados demonstraram que a disposição entre o plano desejado e a posição de varredura com LST pode influenciar na estabilidade da determinação dos parâmetros, uma vez que foram verificados desvios-padrão piores nos parâmetros derivados das varreduras oblíquas.

Cabe ressaltar que a qualidade dos dados derivados de levantamentos com LST está condicionada também à fatores externos, como as propriedades da superfície varrida e as condições ambientais realização. Recomenda-se a continuação das investigações destes fatores na parametrização, a ponderação das variações climáticas (como pressão e temperatura), o teste de significância dos parâmetros determinados, a determinação 
das inclinações dos planos a partir dos parâmetros, o uso de superfícies com diferentes propriedades, ponderando a influência destas sob o ruídos da medição, avaliar os efeitos da existência de possíveis outliers na determinação do plano e comparar o desempenho da utilização do teste de congruência global em relação ao teste Qui-quadrado.

\section{Agradecimentos}

O presente trabalho foi realizado com apoio da Coordenação de Aperfeiçoamento de Pessoal de Nível Superior (CAPES) - Código de Financiamento 001 - e do Conselho Nacional de Desenvolvimento Científico e Tecnológico (CNPq) - Código de Financiamento 141133/2020-1.

\section{Contribuição dos Autores}

A metodologia do trabalho foi definida em conjunto entre os autores. O autor Samir de Souza Oliveira Alves participou na conceptualização, curadoria dos dados, na programação e redação. Os autores Felipe Andrés Carvajal Rodríguez, Lívia Faria Sampaio e Leandro Ítalo Barbosa de Medeiros participaram na curadoria dos dados, na programação e análise formal. Os autores Luís Augusto Koenig Veiga e Ivandro Klein participaram na análise formal, supervisão, investigação e redação.

\section{Conflitos de Interesse}

Os autores declaram que não há conflitos de interesse.

\section{Referências}

AYDIN, C. Power of Global Test in Deformation Analysis. Journal of Surveying Engineering, v. 138, n. 2, p. 51-56, maio 2012. DOI.: 10.1061/(asce)su.1943-5428.0000064.

BARBARELLA, M.; D’AMICO, F.; BLASIIS, M. de; BENEDETTO, A. di; FIANI, M. Use of Terrestrial Laser Scanner for Rigid Airport Pavement Management. Sensors, v. 18, n. 2, p. 44, dez. 2017. DOI.: $10.3390 / \mathrm{s} 18010044$.

BOULOS, P.; CAMARGO, I. de. Geometria Analítica: um tratamento vetorial. $2^{\circ}$ ed. São Paulo: MacGrawHill, 1987.

ERDÉLYI, J.; KOPÁčIK, A.; LIPTÁK, I.; KYRINOVIč, P. Automation of point cloud processing to increase the deformation monitoring accuracy. Applied Geomatics, v. 9, n. 2, p. 105-113, abr. 2017. DOI.: 10.1007/s12518-017-0186-y.

FRÖHLICH, C.; METTENLEITER, M. Terrestrial laser scanning: new perspectives in 3D surveying. In: INTERNATIONAL ARCHIVES OF PHOTOGRAMMETRY, REMOTE SENSING AND SPATIAL INFORMATION SCIENCES, 36., 2004, Freiburg. Proceedings... Freiburg: Institute for Forest Growth, 2004. p. 7-13.

ROFATTO, V. F.; MATSUOKA, M. T.; KLEIN, I.; VERONEZ, M. R.; BONIMANI, M. L.; LEHMANN, R. A half-century of Baarda's concept of reliability: a review, new perspectives, and applications: a review, new perspectives, and applications. Survey Review, v. 52, n. 372, p. 261-277, nov. 2018. DOI.: 10.1080/00396265.2018.1548118.

GEMAEL, C.; MACHADO, A. M. L.; WANDRESEN, R. Introdução ao ajustamento de observações. $2^{a}$ ed. Curitiba: Ed. UFPR. 2015.

GHILANI, Charles D. Adjustment Computations: Spatial Data Analysis. $6^{\text {a }}$ ed. Hoboken, New Jersey: John Wiley \& Sons, Inc. 2018.

GONÇALVES, E. M. Planejamento de rede de monitoramento voltada para o monitoramento de 
estruturas empregando-se LST (laser scanner terrestre). 104 f. Dissertação (Mestrado em Ciências Geodésicas) - Departamento de Geomática, Setor de Ciências da Terra, Universidade Federal do Paraná, Curitiba, 2018.

HOLST, C.; KUHLMANN, H. Challenges and Present Fields of Action at Laser Scanner Based Deformation Analyses. Journal of Applied Geodesy, v. 10, n. 1, p. 17-25, jan. 2016. DOI.: 10.1515/jag-2015-0025.

JAAFAR, H. A.; MENG, X.; SOWTER, A. Terrestrial laser scanner error quantification for the purpose of monitoring. Survey Review, v. 50, n. 360, p. 232-248, 7 mar. 2017. DOI.: 10.1080/00396265.2016.1259721.

JERKE, A. Análise do Monitoramento Geodésico da Barragem da Usina Hidrelétrica Mauá com Equipamentos de Diferentes Precisões e Diferentes Softwares de Processamento. 110 f. Dissertação (Mestrado em Ciências Geodésicas) - Departamento de Geomática, Setor de Ciências da Terra, Universidade Federal do Paraná, Curitiba, 2019.

KLEIN, I. Controle de Qualidade no Ajustamento de Observações Geodésicas. 321 f. Dissertação (Mestrado em Sensoriamento Remoto) - Departamento de Geodésia, Universidade Federal do Rio Grande do Sul, Porto Alegre, 2011.

LEICA. Leica Geosystems. Leica BLK360: Manual do Usuário. 2ª ed. Brasil, 2018.

LENARTOVICZ, I. R. Avaliação da potencialidade do laser scanner terrestre no monitoramento de estruturas: estudo de caso UHE Mauá. 123 f. Dissertação (Mestrado em Ciências Geodésicas) Departamento de Geomática, Setor de Ciências da Terra, Universidade Federal do Paraná, Curitiba, 2013.

LIMA, E. L. Geometria analítica e álgebra linear. $1^{\text {a }}$ ed. Rio de Janeiro: IMPA, 2014.

LINDENBERGH, R.; PFEIFER, N. A statistical deformation analysis of two epochs of terrestrial laser data of a lock. In: CONFERENCE ON OPTICAL 3D MEASUREMENT TECHNIQUES, 7., 2005, Vienna. Proceedings... Vienna, 2005. p. 61-70.

Lovas, T.; Barsi, A.; Detrekoi, A.; Dunai, L.; Csak, Z.; Polgar, A.; Berenyi, A.; Kibedy, Z.; $\quad$ Szocs, K. Terrestrial laser scanning in deformation measurements of structures. In: INTERNATIONAL ARCHIVES OF PHOTOGRAMMETRY, REMOTE SENSING AND SPATIAL INFORMATION SCIENCES, 36., 2008, Pequim. Proceedings... Pequim, 2008, p. 527-532.

MONSERRAT, O.; CROSETTO, M. Deformation measurement using terrestrial laser scanning data and least squares 3D surface matching. ISPRS Journal of Photogrammetry and Remote Sensing, v. 63, n. 1, p. 142-154, jan. 2008. DOI.: 10.1016/j.isprsjprs.2007.07.008.

PETRIE, G.; TOTH, C. K. Topographic Laser Ranging and Scanning: Principles and Processing. $2^{\mathrm{a}}$ ed. Boca Raton: Taylor \& Francis Group, 2018.

SCHMITZ, B.; HOLST, C.; MEDIC, T.; LICHTI, D.; KUHLMANN, H. How to Efficiently Determine the Range Precision of 3D Terrestrial Laser Scanners. Sensors, v. 19, n. 6, p. 1466, 26 mar. 2019. DOI.: $10.3390 / \mathrm{s} 19061466$.

SCHNEIDER, D. Terrestrial laser scanning for area-based deformation analysis of towers and water damns. In: FIG SYMPOSIUM ON DEFORMATION MEASUREMENT, 12., 2006, Baden. Proceedings... Baden, 2006. p. 1-10.

GENECHTEN, B. V. Theory and practice on Terrestrial Laser Scanning: Training material based on practical applications. Valência, 2008.

VENTURI, J. J. Álgebra Vetorial e Geometria Analítica. 10 a ed. Curitiba: Gráfica Infante, 2015.

VOSSElmAN, G.; MAAS, H. Airborne and Terrestrial Laser Scanning. $1^{\mathrm{a}}$ ed. Dunbeath: Whittles Publishing, 2010.

WALTON, G.; DELALOYE, D.; DIEDERICHS, M. S. Development of an elliptical fitting algorithm to improve change detection capabilities with applications for deformation monitoring in circular tunnels and shafts. Tunnelling and Underground Space Technology, v. 43, p. 336-349, jul. 2014. DOI.: 10.1016/j.tust.2014.05.014. 


\section{Biografia do autor principal}

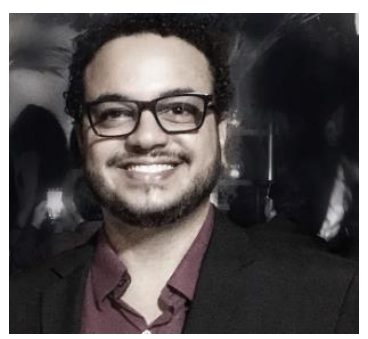

Samir de Souza Oliveira Alves, natural do Rio de Janeiro-RJ. Engenheiro Agrimensor e Cartógrafo formado pela Universidade Federal Rural do Rio de Janeiro. Mestre em Ciências Geodésicas pelo Programa Pós-Graduação em Ciências Geodésicas da UFPR. Pesquisador de nível doutorado do Laboratório de Geodésia Aplicada à Engenharia da UFPR, com ênfase no desenvolvimento de metodologias de aplicações de laser scanner terrestre em levantamentos geodésicos de estruturas e patrimônios históricos. devido crédito pela criação original. 